\title{
Displacement-based finite difference approximations of derivatives of the tangent stiffness matrix with respect to the load parameter
}

\author{
Xin Jia ${ }^{1, *}$ and Herbert A. Mang ${ }^{1}$ \\ ${ }^{1}$ Institute for Mechanics of Materials and Structures, Vienna University of Technology, Vienna, Austria.
}

\begin{abstract}
The vehicle to investigate to which extent energy-based categorization of buckling can be linked up with spherical geometry is the so-called consistently linearized eigenproblem. This investigation requires computation of the first and the second derivative of the tangent stiffness matrix $\tilde{\mathbf{K}}_{T}$ with respect to a dimensionless load parameter $\lambda$ in the frame of the Finite Element Method (FEM). A finite-difference approximation of the first derivative of $\tilde{\mathbf{K}}_{T}$, redefined as a directional derivative, has proved to meet the requirements of computational efficiency and sufficient accuracy. It represents a displacement-based finite-difference approximation, abbreviated as DBFDA. The present work is devoted to the computation of a DBFDA of the second derivative of $\tilde{\mathbf{K}}_{T}$ with respect to $\lambda$. For the special case of a two-dimensional co-rotational beam element, an analytical solution of this derivative is presented. A circular arch, subjected to a vertical point load on its apex, serves as an example for numerically assessing the usefulness of the computed DBFDAs of the first and the second derivative of $\tilde{\mathbf{K}}_{T}$ with respect to $\lambda$.
\end{abstract}

Copyright line will be provided by the publisher

\section{Introduction}

The tangent stiffness matrix $\tilde{\mathbf{K}}_{T}$ and its derivatives with respect to $\lambda$, denoted as $\dot{\tilde{\mathbf{K}}}_{T}$, and $\ddot{\tilde{\mathbf{K}}}_{T}, \ldots$, play an important role, e.g., in sensitivity analyses of the initial postbuckling behavior of structures [1]. In this paper, displacement-based finite difference approximations (DBFDA) of $\dot{\tilde{\mathbf{K}}}_{T}$ and $\ddot{\tilde{\mathbf{K}}}_{T}$ were derived and compared to load-based finite difference approximations (LBFDA), taking the results from the analytical solution as the benchmark results.

\section{Displacement-based finite difference approximations of $\dot{\tilde{\mathbf{K}}}_{T}$ and $\ddot{\tilde{\mathbf{K}}}_{T}$}

$\dot{\tilde{\mathbf{K}}}_{T}:=d \tilde{\mathbf{K}}_{T} / d \lambda$ can be approximated by $\left(\tilde{\mathbf{K}}_{T}(\lambda+h)-\tilde{\mathbf{K}}_{T}(\lambda)\right) / h$ where $h$ is a small change of $\lambda$. Alternatively, based on a directional derivative, given as [1]

$$
\dot{\tilde{\mathbf{K}}}_{T}:=\mathbf{K}_{T, \mathbf{q}} \cdot \dot{\mathbf{q}}=\left.\frac{d}{d h}\right|_{h=0} \mathbf{K}_{T}(\mathbf{q}+h \dot{\mathbf{q}}),
$$

where $\mathbf{q}$ describes the displacement vector, and $\dot{\mathbf{q}}$ is the so-called displacement rate,

$$
\dot{\tilde{\mathbf{K}}}_{T} \approx \frac{\mathbf{K}_{T}(\mathbf{q}+h \dot{\mathbf{q}})-\tilde{\mathbf{K}}_{T}(\mathbf{q})}{h}
$$

where $h$ is a small positive number, and $h \dot{\mathbf{q}}$ describes a small change of the displacement vector in the direction of the displacement rate $\dot{\mathbf{q}}$. Similarly, the DBFDA of $\ddot{\tilde{\mathbf{K}}}_{T}$ can be obtained as

$$
\ddot{\tilde{\mathbf{K}}}_{T} \approx \frac{\mathbf{K}_{T}(\mathbf{q}+2 h \dot{\mathbf{q}}+h \ddot{\mathbf{q}})-2 \mathbf{K}_{T}(\mathbf{q}+h \dot{\mathbf{q}})+\tilde{\mathbf{K}}_{T}(\mathbf{q})}{h^{2}} .
$$

\section{Analytical expressions for $\dot{\tilde{\mathbf{K}}}_{T}$ and $\ddot{\tilde{\mathbf{K}}}_{T}$ based on a co-rotational beam element}

In the frame of the FEM, $\dot{\tilde{\mathbf{K}}}_{T}$ and $\ddot{\tilde{\mathbf{K}}}_{T}$ can be obtained by assembling $\dot{\tilde{\mathbf{K}}}_{T}^{e}$ and $\ddot{\tilde{\mathbf{K}}}_{T}^{e}$, respectively. Herein, $\tilde{\mathbf{K}}_{T}^{e}$ denotes the element tangent stiffness matrix. For a specific element type, i.e. a co-rotational beam element, the analytical expression of

\footnotetext{
* Corresponding author: e-mail xin.jia@tuwien.ac.at, phone +43158801 20253, fax +4315880120299
} 
$\dot{\tilde{\mathbf{K}}}_{T}^{e}$ can be derived. It is given in [2]. The respective expression for $\ddot{\tilde{\mathbf{K}}}_{T}^{e}$ is obtained as follows:

$$
\begin{aligned}
& \dot{\mathbf{K}}_{T}^{e}=\mathbf{X}^{T} \ddot{\mathbf{K}}_{T}^{e} \mathbf{X}+2 \dot{\mathbf{X}}^{T} \dot{\mathbf{K}}_{T}^{e} \mathbf{X}+2 \dot{\mathbf{X}}^{T} \overline{\mathbf{K}}_{T}^{e} \dot{\mathbf{X}}+\ddot{\mathbf{X}}^{T} \overline{\mathbf{K}}_{T}^{e} \mathbf{X}+\mathbf{X}^{T} \overline{\mathbf{K}}_{T}^{e} \ddot{\mathbf{X}}+2 \mathbf{X}^{T} \dot{\mathbf{K}}_{T}^{e} \dot{\mathbf{X}}+ \\
& \frac{\mathbf{z z}^{T} \ddot{\bar{N}}+2 \dot{\mathbf{z}} \dot{\mathbf{z}}^{T} \bar{N}+\ddot{z}^{T} \bar{N}+2 \dot{z z}^{T} \dot{\bar{N}}+\ddot{\mathbf{z}} \mathbf{z}^{T} \bar{N}+2 \dot{\mathbf{z}} \mathbf{z}^{T} \dot{\bar{N}}}{\hat{l}}+\frac{2\left(\dot{\mathbf{r}} \mathbf{z}^{T}+\mathbf{r} \dot{\mathbf{z}}^{T}+\dot{\mathbf{z}} \mathbf{r}^{T}+\dot{\mathbf{z}}^{T}\right)\left(\dot{\bar{M}}_{1}+\dot{\bar{M}}_{2}\right)}{\hat{l}^{2}}+ \\
& \frac{\left(\ddot{\mathbf{r}} \mathbf{z}^{T}+2 \dot{\mathbf{r}}^{T}+\mathbf{r} \ddot{\mathbf{z}}^{T}+2 \dot{\mathbf{z}} \dot{\mathbf{r}}^{T}+\mathbf{z} \ddot{\mathbf{r}}^{T}\right)\left(\bar{M}_{1}+\bar{M}_{2}\right)}{\hat{l}^{2}}+\frac{\left(\mathbf{r} \mathbf{z}^{T}+\mathbf{z} \mathbf{r}^{T}\right)\left(\ddot{\bar{M}}_{1}+\ddot{\bar{M}}_{2}\right)-2 \dot{\mathbf{z}} \mathbf{z}^{T} \bar{N} \dot{\hat{l}}-2 \mathbf{z} \dot{\mathbf{z}}^{T} \bar{N} \dot{\hat{l}}}{\hat{l}^{2}}+ \\
& \frac{-\mathbf{z z}^{T} \bar{N} \ddot{\hat{l}}-2 \mathbf{z z}^{T} \dot{\bar{N}} \dot{\hat{l}}}{\hat{l}^{2}}+\frac{-\left(\mathbf{r} \mathbf{z}^{T}+\mathbf{z r}^{T}\right)\left(\bar{M}_{1}+\bar{M}_{2}\right) \ddot{\hat{l}}-4\left(\dot{\mathbf{r}} \mathbf{z}^{T}+\mathbf{r} \dot{\mathbf{z}}^{T}+\dot{\mathbf{z}} \mathbf{r}^{T}+\mathbf{z i}^{T}\right)\left(\bar{M}_{1}+\bar{M}_{2}\right) \dot{\hat{l}}}{\hat{l}^{3}}+ \\
& \frac{-4\left(\mathbf{r z} \mathbf{z}^{T}+\mathbf{z r}^{T}\right)\left(\dot{\bar{M}}_{1}+\dot{\bar{M}}_{2}\right) \dot{\hat{l}}+2 \mathbf{z z}^{T} \bar{N} \ddot{\hat{l}}}{\hat{l}^{3}}+\frac{6\left(\mathbf{r z}^{T}+\mathbf{z r}^{T}\right)\left(\bar{M}_{1}+\bar{M}_{2}\right) \ddot{\hat{l}}}{\hat{l}^{4}}
\end{aligned}
$$

where $\mathbf{X}$ denotes the matrix for transformations from local to global coordinates; $\hat{l}$ denotes the length of the deformed beam; $\mathbf{r}, \mathbf{z}$ are vectors used for abbreviation of lengthy expressions; $\overline{\mathbf{K}}_{T}^{e}$ is the element tangent stiffness matrix in the local coordinate system; $\bar{N}, \bar{M}_{1}, \bar{M}_{2}$ denote the components of the force vector in the local coordinate system. This approach depends on the chosen element. Therefore, it is impractical in view of the great number of types of finite elements and the large variety of technical problems, requiring the choice of one or more problem-specific elements.

\section{Numerical verification}

Fig.1a shows a circular arch subjected to a vertical point load $\bar{P}$ at the vertex. The geometric properties and the material parameters are also shown in this figure. The arch is discretized by 100 two-node beam elements. It is analyzed by means of NFEM-CRB2D, which is a matlab-based nonlinear finite element program developed by the first author. The quality of the
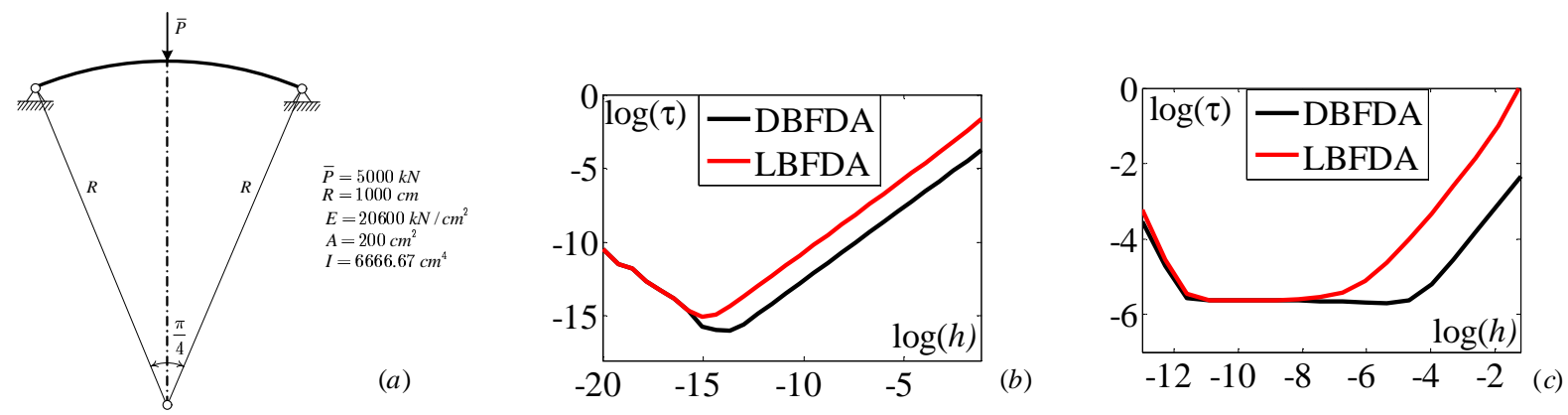

Fig. 1: Numerical example-circular arch: (a) configuration and design parameters, (b) error assessment of $\dot{\tilde{\mathbf{K}}}_{T}$, (c) error assessment of $\ddot{\tilde{\mathbf{K}}}_{T}$.

DBFDA and LBFDA of $\dot{\tilde{\mathbf{K}}}_{T}$ and $\ddot{\tilde{\mathbf{K}}}_{T}$ is assessed by comparing the dependence of the error of $\dot{\tilde{\mathbf{K}}}_{T}$ and of $\ddot{\tilde{\mathbf{K}}}_{T}$ on $h$ (see Fig.1b and Fig.1c). In Fig.1b

$$
\tau:=\frac{\left\|\dot{\tilde{\mathbf{K}}}_{T-D B F D A(L B F D A)}-\dot{\tilde{\mathbf{K}}}_{T-E X}\right\|}{\left\|\dot{\tilde{\mathbf{K}}}_{T-E X}\right\|} .
$$

where $\dot{\tilde{\mathbf{K}}}_{T-E X}$ indicates calculation of this matrix from the analytical expression derived in Section 3, and $\dot{\tilde{\mathbf{K}}}_{T-D B F D A(L B F D A)}$ refers to calculation of this matrix by means of the the DBFDA and LBFDA, respectively. By observing Fig.1bc, it is seen that (a) the convergence rate of the DBFDA of $\dot{\tilde{\mathbf{K}}}_{T}\left(\ddot{\widetilde{\mathbf{K}}}_{T}\right)$ is the same as that of the LBFDA of $\dot{\tilde{\mathbf{K}}}_{T}\left(\ddot{\widetilde{\mathbf{K}}}_{T}\right)$; (b) the DBFDA of $\dot{\tilde{\mathbf{K}}}_{T}\left(\ddot{\tilde{\mathbf{K}}}_{T}\right)$ is more accurate than the LBFDA of $\dot{\tilde{\mathbf{K}}}_{T}\left(\ddot{\tilde{\mathbf{K}}}_{T}\right)$.

\section{References}

[1] H. A. Mang, C. Schranz, and P. Mackenzie-Helnwein, Conversion from imperfection-sensitive into imperfection-insensitive elastic structures I: Theory, Computer Methods in Applied Mechanics and Engineering, 195(13-16), 1422-1457, 2006.

[2] X. Jia, and H. A. Mang, Determination of the derivative of the tangent stiffness matrix with respect to the load parameter, PAMMProceedings in Applied Mathematics and Mechanics, 13, 119-120, 2013. 